\title{
Long X-ray flares from the central source in RCW 103
}

\section{XMM-Newton and VLT observations in the aftermath of the 2016 outburst}

\author{
P. Esposito ${ }^{1}$, A. De Luca ${ }^{1,2}$, R. Turolla ${ }^{3,4}$, F. Coti Zelati5 ${ }^{5,6}$, W. Hummel ${ }^{7}$, A. Tiengo ${ }^{8,1,2}$, G. L. Israel ${ }^{9}$, N. Rea ${ }^{5,6}$, \\ R. P. Mignani ${ }^{1,10}$, and A. Borghese ${ }^{11}$
}

1 INAF-Istituto di Astrofisica Spaziale e Fisica Cosmica di Milano, Via A. Corti 12, 20133 Milano, Italy e-mail: paolo.esposito@inaf.it

2 INFN-Istituto Nazionale di Fisica Nucleare, Sezione di Pavia, Via A. Bassi 6, 27100 Pavia, Italy

3 Dipartimento di Fisica e Astronomia "Galileo Galilei”, Università di Padova, Via F. Marzolo 8, 35131 Padova, Italy

${ }^{4}$ Mullard Space Science Laboratory, University College London, Holmbury St. Mary, Dorking, Surrey RH5 6NT, UK

5 Institute of Space Sciences (ICE, CSIC), Campus UAB, Carrer de Can Magrans s/n, 08193 Barcelona, Spain

${ }^{6}$ Institut d'Estudis Espacials de Catalunya (IEEC), Gran Capità 2-4, 08034 Barcelona, Spain

7 European Southern Observatory, Karl-Schwarzschild-Straße 2, 85748 Garching bei München, Germany

8 Scuola Universitaria Superiore IUSS Pavia, Piazza della Vittoria 15, 27100 Pavia, Italy

9 INAF-Osservatorio Astronomico di Roma, Via Frascati 33, 00078 Monteporzio Catone, Italy

10 Janusz Gil Institute of Astronomy, University of Zielona Góra, ul. Szafrana 2, 65-516 Zielona Góra, Poland

11 Anton Pannekoek Institute for Astronomy, University of Amsterdam, Science Park 904, 1098 XH Amsterdam, The Netherlands

Received 6 March 2019 / Accepted 10 April 2019

\begin{abstract}
We observed the slowly revolving pulsar 1E 161348-5055 (1E 1613, spin period of $6.67 \mathrm{~h}$ ) in the supernova remnant RCW 103 twice with XMM-Newton and once with the Very Large Telescope (VLT). The VLT observation was performed on 2016 June 30 , about a week after the detection of a large outburst from 1E 1613. At the position of 1E 1613, we found a near-infrared source with $K_{s}=20.68 \pm 0.12 \mathrm{mag}$ that was not detected $\left(K_{s}>21.2 \mathrm{mag}\right)$ in data collected with the same instruments in 2006, during X-ray quiescence. Its position and behavior are consistent with a counterpart in the literature that was discovered with the Hubble Space Telescope in the following weeks in adjacent near-IR bands. The XMM-Newton pointings were carried out on 2016 August 19 and on 2018 February 14. While the collected spectra are similar in shape between each other and to what is observed in quiescence (a blackbody with $k T \sim 0.5 \mathrm{keV}$ plus a second, harder component, either another hotter blackbody with $k T \sim 1.2 \mathrm{keV}$ or a power law with photon index $\Gamma \sim 3$ ), the two pointings caught $1 \mathrm{E} 1613$ at different luminosity throughout its decay pattern: about $4.8 \times 10^{34} \mathrm{erg} \mathrm{s}^{-1}$ in 2016 and $1.2 \times 10^{34} \mathrm{erg} \mathrm{s}^{-1}$ in $2018(0.5-10 \mathrm{keV}$, for the double-blackbody model and for $3.3 \mathrm{kpc})$, which is still almost about ten times brighter than the quiescent level. The pulse profile displayed dramatic changes, apparently evolving from the complex multipeak morphology observed in high-luminosity states to the more sinusoidal form characteristic of latency. The inspection of the X-ray light curves revealed two flares with unusual properties in the 2016 observation: they are long ( $\sim \mathrm{ks}$ to be compared with $0.1-1 \mathrm{~s}$ of typical magnetar bursts) and faint $\left(\approx 10^{34} \mathrm{erg} \mathrm{s}^{-1}\right.$, with respect to $10^{38} \mathrm{erg} \mathrm{s}^{-1}$ or more in magnetars). Their spectra are comparatively soft and resemble the hotter thermal component of the persistent emission. If the flares and the latter component have a common origin, this may be a spot on the star surface that is heated by back-flowing currents that are induced by a magnetospheric twist. In this hypothesis, since the increase in luminosity of $1 \mathrm{E} 1613$ during the flare is only $20 \%$, an irregular variation of the same order in the twist angle could account for it.
\end{abstract}

Key words. X-rays: individuals: 1E 161348-5055 - stars: neutron

\section{Introduction}

The X-ray source 1E 161348-5055 (1E 1613) lies at the center of the young ( $2 \mathrm{kyr}$ ) supernova remnant (SNR) RCW 103. It was discovered with Einstein and was suggested to be a radio-quiet isolated neutron star (NS) by Tuohy \& Garmire (1980). Over the years, the behavior of 1E 1613 has set it apart from any other compact object source (De Luca 2008, 2017): the source displays a strong variability on scales of months or years (a large outburst with an increase in luminosity from $\sim 3 \times 10^{33}$ to over $3 \times 10^{35} \mathrm{erg} \mathrm{s}^{-1}$ occurred in 1999), and features a modulation in flux with a period of $6.67 \mathrm{~h}$, together with dramatic changes in the pulse profile (which are correlated with the flux level). Considering also the young age of 1E 1613 and the lack of an optical or IR counterpart, De Luca et al. (2006) discussed two main possibilities: 1E 1613 could be either a very young lowmass X-ray binary (LMXB, the first observed inside an SNR, see also Bhadkamkar \& Ghosh 2009), or an isolated magnetar that slowly revolves at an abnormal period of $6.67 \mathrm{~h}$, possibly due to a propeller interaction with a fallback disk (see also $\mathrm{Li}$ 2007; Popov et al. 2015). Even more exotic pictures have been proposed, such as an LMXB with a supermagnetic NS locked in synchronous rotation with the orbit (Pizzolato et al. 2008), or an evolved Thorne-Żytkow object (Liu et al. 2015).

Recently, a remarkable event added new elements to the decade-long enigma. On 2016 June 22, the Burst Alert Telescope (BAT) on board the Neil Gehrels Swift Observatory detected a short X-ray burst from the direction of RCW 103 
(Rea et al. 2016; D'Aì et al. 2016). The light curve of the short burst $(\sim 10 \mathrm{~ms})$ shows a double-peak profile, the spectrum of which is well described by a blackbody model $(k T \sim$ $9 \mathrm{keV}$ ) and a luminosity of $\sim 2 \times 10^{39} \mathrm{erg} \mathrm{s}^{-1}$ (for a distance of $3.3 \mathrm{kpc}$; Caswell et al. 1975; Rea et al. 2016). All in all, the event was a signature magnetar burst (Turolla et al. 2015; Kaspi \& Beloborodov 2017; Esposito et al. 2018). Concurrently, an enhancement by a factor $>100$ in the X-ray flux of $1 \mathrm{E} 1613$ with respect to the quiescent level (observed for several years and up to one month before) was measured with the Swift X-Ray Telescope (XRT). On 2016 June 25, Chandra and NUSTAR observations showed a flux modulation at the known $6.67 \mathrm{~h}$ period with two main peaks per cycle, which is different from the nearly sinusoidal shape seen during the quiescent state of the source (Esposito et al. 2011) and 1E 1613 was detected for the first time at hard X-rays, up to $\sim 30 \mathrm{keV}$ (Rea et al. 2016).

An intense multi-instrument monitoring showed that after more than $1 \mathrm{yr}$ since the onset of the outburst, 1E 1613 was still about ten times brighter than usual and that the total energy emitted $\left(\sim 2 \times 10^{42} \mathrm{erg}\right)$, the flux decay pattern, and the spectral evolution were similar to what is generally observed in magnetars (Rea et al. 2016; Borghese et al. 2018; Coti Zelati et al. 2018). Moreover, in HST images taken on 2016 July 4 and August 11, Tendulkar et al. (2017) detected a likely IR counterpart with $J$ magnitude 26.3 and $H$ magnitude 24.2, implying a minimum brightening of at least $1.3 \mathrm{mag}(H)$ compared to the non-detections in the previous (2002) observations (with a different instrument, De Luca et al. 2008) and an X-ray-to-IR luminosity ratio consistent with typical values or limits for magnetars and isolated NSs (Mignani 2011; Olausen \& Kaspi 2014). Finally, the optical counterpart rules out for 1E 1613 all binary scenarios in which the IR emission comes from an accretion disk or from a stellar companion (non-degenerate or white dwarf).

In this paper, we report on the results from the analysis of two XMM-Newton exposures carried out in 2016 August and 2018 February that seized the source at two different flux levels along its decay pattern. We also present VLT/NAOS+CONICA (NaCo) IR images taken at Paranal in the night of 2016 June 29-30, a few days before the HST observations that discovered the counterpart of 1E 1613 (Tendulkar et al. 2017). We detected a faint source that can be identified to be that of Tendulkar et al. (2017), which was not detected in previous $\mathrm{NaCo}$ data that were collected in 2006 (De Luca et al. 2008), while 1E 1613 was in quiescence.

\section{Very Large Telescope observations and results}

We performed a target of opportunity observation (Program ID: 297.D-5042(A)) of the field of 1E 1613 on 2016 June 30 at the ESO Paranal Observatory with NaCo (Lenzen et al. 2003; Rousset et al. 2003), the adaptive optics imager and spectrometer mounted at the VLT Unit 1 (Antu). We adopted the same setup as we used in our previous NaCo observations of 1E 1613 (2006 May; Program ID: 077.D-0764(A); De Luca et al. 2008). The instrument was operated with the $\mathrm{S} 27$ camera, giving a field of view of $28^{\prime \prime} \times 28^{\prime \prime}$ and a pixel scale of $00^{\prime} 027$. The visual (VIS) dichroic element and wave-front sensor (4500-10000 $\AA$ ) were used. Observations were performed in the $K_{s}$ filter $(\lambda=2.18 \mu \mathrm{m}$; $\Delta \lambda=0.35 \mu \mathrm{m}$ FWHM). The only suitable reference star for the adaptive optics correction is the GSC-2 star S230213317483 ( $V \sim 15.2$ ), located 21'.'1 away from our target. We performed three observations, lasting $2500 \mathrm{~s}$ each, split into sequences of short dithered exposures with $50 \mathrm{~s}$ integration. At variance with our 2006 observations, the target region was located close to the center of quadrant 4 to keep it away from the malfunctioning quadrant $2^{1}$ in the dithering pattern; this resulted in a $\sim 10^{\prime \prime}$ shift of the target within the field of view with respect to the 2006 data. Airmass was in the 1.12-1.17 range; seeing conditions were very good, in the $0.30-0 . ' 50$ range, mostly below $0 . ' 40$. Sky conditions were mostly photometric. Night (twilight flat fields) and day-time calibration frames (darks, lamp flat fields) were taken daily as part of the $\mathrm{NaCo}$ calibration plan. The data were processed using the ESO NaCo pipeline, and the science images were coadded using the eclipse software (Devillard 1997) to produce a master image with 7500 s exposure time.

Our goal is to search for sources in the error region of $1 \mathrm{E} 1613$ that display variability with respect to our 2006 observations. Thus, the source catalog produced by De Luca et al. (2008) was adopted as a reference for both astrometry and photometry. We ran a source detection on our master image using the SExtractor software (Bertin \& Arnouts 1996). Using a set of $\sim 300$ sources down to $K_{s}=20$, we superimposed the master image on the image obtained in 2006 with a root mean square (rms) $<0$.'01. Using the same set of stars, we fit the zero-point needed to convert the instrumental aperture magnitudes to $K_{s}$ magnitudes as computed in our previous work (De Luca et al. 2008). This exercise yields a rather large scatter in the flux measurements between the two epochs, with an rms of $\sim 0.25 \mathrm{mag}$. We noted that residuals clearly depend on the position of the sources within the field of view. This could be due to anisoplanatic effects (i.e., related to the change in point spread function as a function of distance from the guide star in adaptive optics observations) or to the positioning of the target in different quadrants. As a simple approach, we added a correction to the zero-point that was linearly dependent on the distance to the guide star. The fit results were much better, with an rms below 0.10 mag on the whole field of view.

Inspection of the master image shows a new source that is located close to the center of the error region for $1 \mathrm{E} 1613$. This was not detected in our 2006 data (see Fig. 1). Its position is RA $=16^{\mathrm{h}} 17^{\mathrm{m}} 36^{\mathrm{s}} .21$, Dec $=-51^{\circ} 02^{\prime} 24^{\prime \prime} .6(\mathrm{~J} 2000)$, with an uncertainty of $\sim 00^{\prime} 1$ per coordinate (dominated by the uncertainty in the astrometric solution of the $2006 \mathrm{NaCo}$ image that was used as a reference; De Luca et al. 2008) and its magnitude is $K_{s}=20.68 \pm 0.12$. All of the seven sources mentioned by De Luca et al. (2008) as candidate counterparts are also clearly detected in the new image, with no large flux variations with respect to the values measured in 2006 ( $\mathrm{rms}$ of $\sim 0.13 \mathrm{mag}$ ). We performed simulations for which we added artificial sources to the $2006 \mathrm{NaCo}$ image at the position of the new source detected in 2016. We estimate that a source at the 2016 magnitude level would have been easily detected, with a signal-to-noise ratio of $\sim 4.5$. The $3 \sigma$ upper limit to any undetected source at that position in the 2006 image is $K_{s}>21.2$.

We also investigated a possible short-term variability of the new candidate counterpart of $1 \mathrm{E} 1613$. To this aim, we generated images with $1000 \mathrm{~s}$ exposure time as well as with $2500 \mathrm{~s}$ exposure time by combining 20 and 50 consecutive frames with $50 \mathrm{~s}$ integration time, respectively. For each image, we performed astrometric and photometric calibration as described above for the master image, with similar accuracy. This allowed us to produce light curves with $\sim 1000 \mathrm{~s}$ and $\sim 2500 \mathrm{~s}$ binning for $\sim 380$ and $\sim 900$ sources, respectively, including the new candidate counterpart as well as all of the seven candidate counterparts mentioned by De Luca et al. (2008). As in our previous

1 See the NaCo News Page at http://www.eso.org/sci/ facilities/paranal/instruments/naco/news.html 

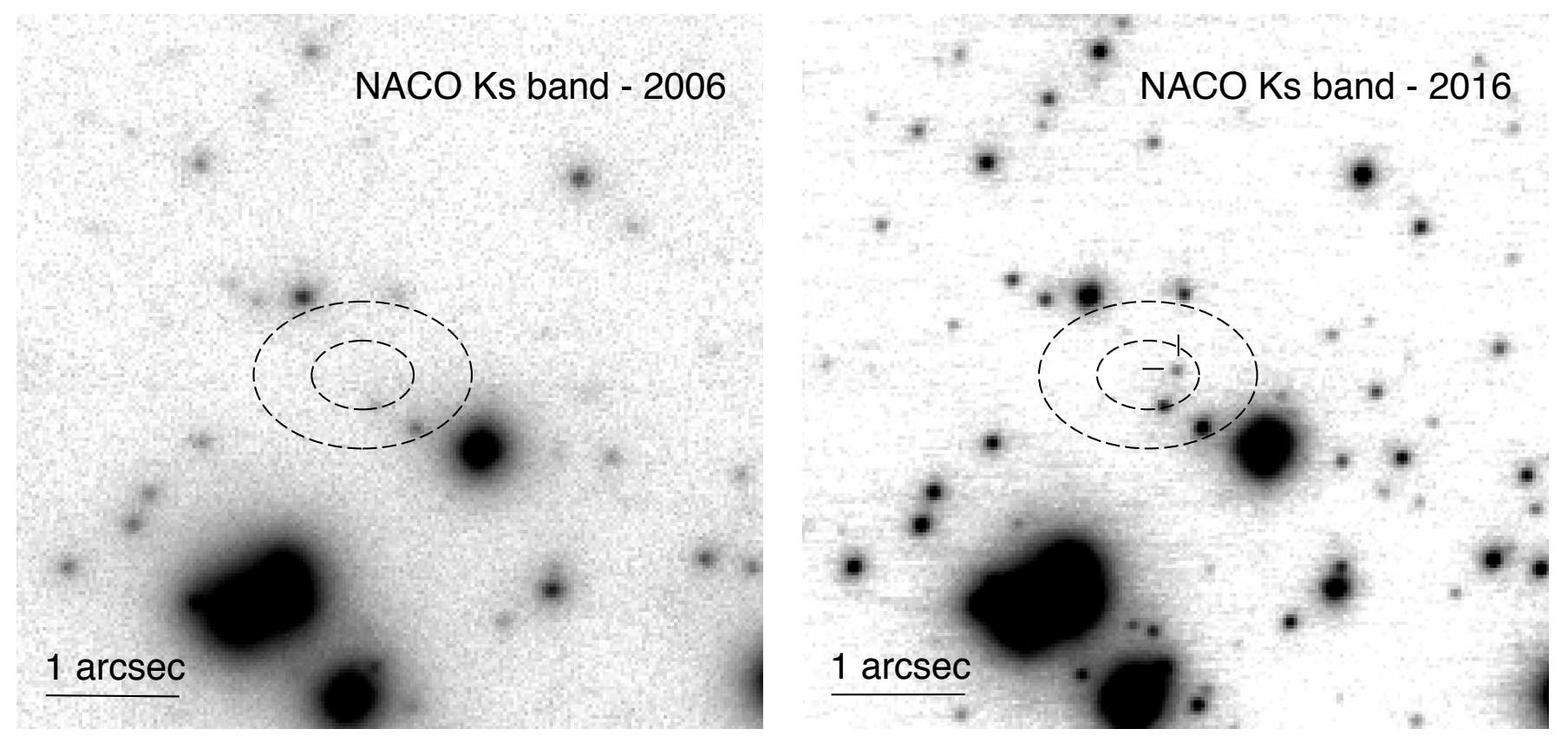

Fig. 1. VLT/NaCo images of the field of 1E 1613 in RCW 103 in 2006 and 2016 . The ellipses show the $68 \%$ and $99 \%$ position uncertainty of 1E 1613. The source detected in 2016 at $K_{s}=20.68 \pm 0.12$ that previously was not detected $\left(K_{s}>21.2\right)$ is marked with two ticks. The same source is labeled " 8 " in Fig. 2 of Tendulkar et al. (2017).

Table 1. XMM-Newton observations. In the second observation, the net exposure times are after the screening for proton flares.

\begin{tabular}{llc}
\hline \hline Obs. ID & Date & $\begin{array}{c}\text { Net exposure (ks) } \\
\text { pn/MOS1/MOS2 }\end{array}$ \\
\hline 0743750201 (A) & $2016-08-19 / 20$ & $56.7 / 76.9 / 76.7$ \\
0805050101 (B) & $2018-02-14$ & $43.8 / 59.1 / 59.6$ \\
\hline
\end{tabular}

analysis (De Luca et al. 2008), we found a larger rms variability for fainter sources, which implies that our photometric measurements are contaminated by random errors (see discussion in De Luca et al. 2008). The new candidate counterpart $\left(K_{s} \sim 20.7\right)$ displays an rms of $\sim 0.22 \mathrm{mag}$ and of $\sim 0.23 \mathrm{mag}$ on the $1000 \mathrm{~s}$ and on the $2500 \mathrm{~s}$ timescales, respectively. These results are broadly consistent with the apparent rms variability for sources in the $K_{s}$ magnitude range $20.0-21.0$, which is of $\sim 0.18 \mathrm{mag}$ and of $\sim 0.13 \mathrm{mag}$ on the $1000 \mathrm{~s}$ (55 sources) and on the $2500 \mathrm{~s}$ timescale (335 sources), respectively. Thus, we cannot draw firm conclusions about a possible modulation of the flux of the new candidate counterpart. The same is true for all of the seven candidate counterparts mentioned by De Luca et al. (2008).

\section{XMM-Newton observations and results}

Two XMM-Newton observations of 1E 1613 were taken after the 2016 outburst, one on 2016 August 19-20 (Obs. ID 0743750201) and one on 2018 February 14 (Obs. ID 0805050101). The 2016 exposure lasted about $82.5 \mathrm{ks}$, and the EPIC pn (Strüder et al. 2001) was operated in Small Window mode (time resolution: $5.7 \mathrm{~ms}$ ) and the MOS detectors (Turner et al. 2001) in Large Window mode (time resolution: $0.9 \mathrm{~s})$; all cameras mounted the thin optical-blocking filter. The exposures of the MOS cameras were interrupted after $\sim 0.5 \mathrm{ks}$ and later resumed, with a gap of $\sim 3.2 \mathrm{ks}$ both in the MOS1 and MOS2 data. The 2018 pointing was $63 \mathrm{ks}$ long and all the detec-

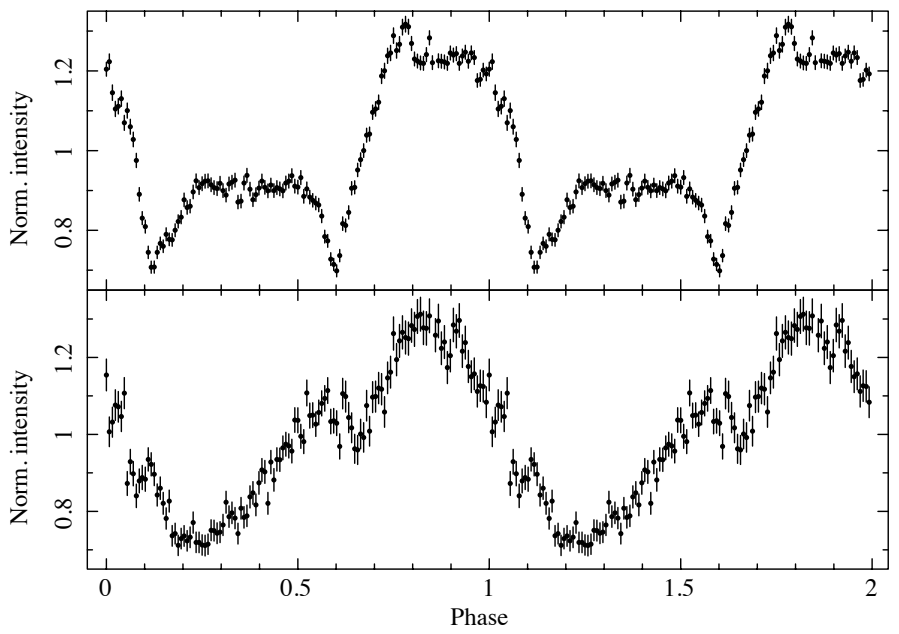

Fig. 2. Folded light curves of 1E 1613. Top panel: 2016 data set (A) and bottom panel: 2018 data set (B). The energy range is $1-10 \mathrm{keV}$, and both data sets were folded with epoch MJD 57619 in the FTOOLS task efold and the period of Esposito et al. (2011, $P=24030.42$ s). The profile apparently evolves from the complex multi-peak structure observed in high flux states to the simpler (nearly sinusoidal) shape observed in quiescence (see Fig. 2 of De Luca et al. 2006, Fig. 3 of Rea et al. 2016, and Fig. 4 of Esposito et al. 2011 for a collection of the pulse profiles that were observed in quiescence).

tors were in Full Frame mode (time resolution: $73.4 \mathrm{~ms}$ for the pn and $2.7 \mathrm{~s}$ for the MOSs), using the thin filter for the pn and the medium filter for the MOSs. The second observation was affected by intervals of flaring particle background, which were removed using intensity filters on the light curves; this screening reduced by $\sim 20 \%$ the net exposure time in the pn and by a lesser amount in the MOS cameras (see Table 1 for more details).

The raw observation data files were processed with the Science Analysis Software (SAS; Gabriel et al. 2004) v.17.1. To extract the event lists and spectra, we used the same regions as 
Table 2. Summary of the spectral results.

\begin{tabular}{|c|c|c|c|c|c|c|c|c|c|}
\hline Obs. & Model & $\begin{array}{c}N_{\mathrm{H}} \\
\left(10^{22} \mathrm{~cm}^{-2}\right)\end{array}$ & $\begin{array}{l}k_{\mathrm{B}} T_{1} \\
(\mathrm{keV})\end{array}$ & $\begin{array}{c}R_{\mathrm{bb} 1}^{(a)} \\
(\mathrm{km})\end{array}$ & $\begin{array}{l}\Gamma / k_{\mathrm{B}} T_{2} \\
(-/ \mathrm{keV})\end{array}$ & $\begin{array}{c}R_{\mathrm{bb} 2}(a) \\
(\mathrm{km})\end{array}$ & $\begin{array}{c}\text { Flux }{ }^{(b)} \\
\left(10^{-11} \mathrm{erg} \mathrm{cm}^{-2} \mathrm{~s}^{-1}\right)\end{array}$ & $\begin{array}{l}\text { Luminosity }{ }^{(b)} \\
\left(10^{34} \mathrm{erg} \mathrm{s}^{-1}\right)\end{array}$ & $\chi_{v}^{2}($ \\
\hline \multirow[t]{2}{*}{ A } & B & $1.10 \pm 0.01$ & 551 & 1. & $1.20=$ & $15 \pm 0.01$ & 2.118 & $4.80 \pm 0.03$ & 1.07 \\
\hline & $\mathrm{B}$ & $1.32^{+}$ & $0.574 \pm 0.002$ & $1.68 \pm 0.01$ & 2.80 & - & 2.126 & 6. & 303) \\
\hline \multirow[t]{2}{*}{ B } & $\mathrm{BB}+\mathrm{BB}$ & $0.98 \pm 0.02$ & $0.527_{-0.007}^{+0.006}$ & $1.04_{-0.02}^{+0.03}$ & $1.14 \pm 0.06$ & $0.09_{-0.02}^{+0.01}$ & 0.529 & $1.20 \pm 0.01$ & $1.07(1255)$ \\
\hline & $\mathrm{BB}+\mathrm{PL}$ & $1.32_{-0.07}^{+0.08}$ & $0.543 \pm 0.003$ & $0.92_{-0.02}^{+0.01}$ & $3.1_{-0.2}^{+0.1}$ & - & $0.532_{-0.002}^{+0.003}$ & $2.01_{-0.03}^{+0.04}$ & $1.09(1255)$ \\
\hline
\end{tabular}

Notes. Uncertainties are given at the $1 \sigma$ confidence level. ${ }^{(a)}$ Radius at infinity assuming a distance of $3.3 \mathrm{kpc} .{ }^{(b)}$ Observed (not corrected for the absorption) flux in the $0.5-10 \mathrm{keV}$ range. The luminosity is in the $0.5-10 \mathrm{keV}$ range for a distance of $3.3 \mathrm{kpc}$.

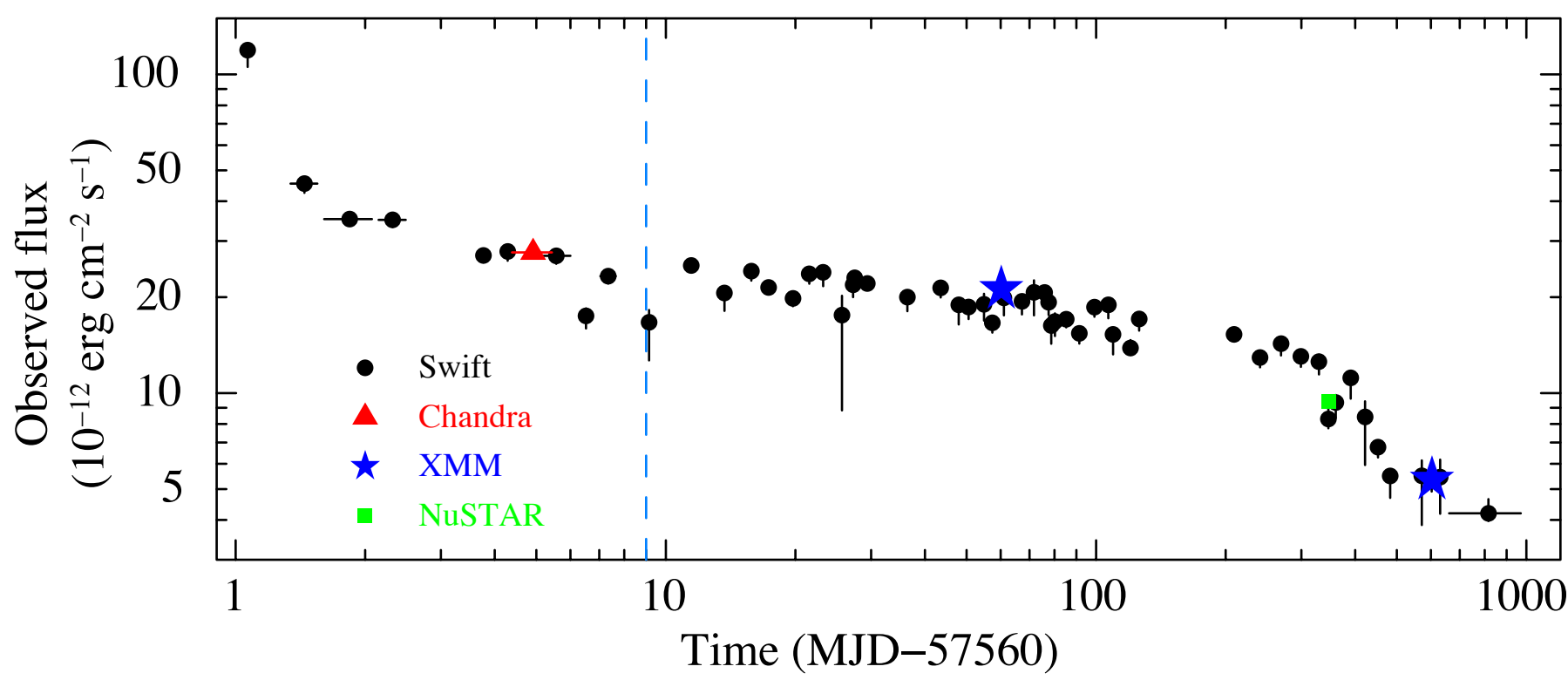

Fig. 3. Long-term light curve of $1 \mathrm{E} 1613$ starting from the 2016 burst. The flux (not corrected for the absorption) is in the $0.5-10 \mathrm{keV}$ band. Except for the XMM-Newton points, the data are from the Magnetar Outburst Online Catalog (MOOC, http://magnetars.ice.csic.es/; Coti Zelati et al. 2018) and the flux values were obtained from a double-blackbody fit. For some data points, the error bar is smaller than the symbol. The dashed line indicates the epoch of the VLT observation.

were selected in De Luca et al. (2006): a circle with a radius of 15 arcsec centered on $1 \mathrm{E} 1613$ and a circle with a radius of 20 arcsec with a center at RA $=16^{\mathrm{h}} 17^{\mathrm{m}} 42^{\mathrm{s}} \cdot 4$, Dec $=-51^{\circ} 02^{\prime} 38^{\prime \prime}$ (J2000), a region where the surface brightness of the RCW 103 SNR is comparable to that of the surroundings of the source, to estimate the background. Spectra were rebinned so as to have a minimum of 30 counts per energy bin, and for each spectrum, the response matrix and ancillary files were generated with the SAS tasks rmfgen and arfgen. To convert the event times into the solar system barycenter, we used the task barycen.

The evolution of the flux, the spectral shape, and the pulse profile of $1 \mathrm{E} 1613$ in the aftermath of the outburst have been studied in detail by Borghese et al. (2018) and Coti Zelati et al. (2018) over a period encompassing the two XMM-Newton observations. Here, we concentrate on the individual data sets.

The $6.67 \mathrm{~h}$ flux modulation is evident in both observations and apparently evolves from the complicate shape that is characteristic of the outbursts toward the nearly sinusoidal profile observed in quiescence (Fig. 2; see also Fig. 2 of De Luca et al. 2006 and Fig. 4 of Esposito et al. 2011). In the first observation, the high flux combined with the long exposure resulted in spectra with hundreds of thousands of source counts $\left(>2 \times 10^{5}\right.$ in the pn and $>10^{5}$ in each MOS). In spectra of such high statistical quality, systematic (calibration) errors are important in the error budget, as can be seen by inspecting the fit residuals at the energies of the effective area edges, where large variations occur rapidly. For this reason we added to the spectra an energy-independent systematic uncertainty of $3 \%$ (see, e.g., Molendi \& Gastaldello 2009).

The spectra may be modeled by a double blackbody or a blackbody-plus-power-law model and an absorption component. For the latter, we adopted the abundances by Wilms et al. (2000) and the Tübingen-Boulder interstellar medium (ISM) absorption model. The parameters derived from the spectral fits are similar to those from previous analyses (De Luca et al. 2006; Rea et al. 2016; Borghese et al. 2018) and are summarized in Table 2.

Figure 3 shows the long-term evolution of the observed flux (the XMM-Newton data refer to the double-blackbody model).

Inspecting the light curves, we realized that they contained unusual features in the first observation. At least two long-lasting flares occurred, the first $\sim 32 \mathrm{ks}$ after the start of the observation, the second at about $78 \mathrm{ks}$ (see Fig. 4). They can be identified because $(>5 \mathrm{keV})$ they appear like spikes in the hard band. The first flare lasted approximately $1.2 \mathrm{ks}$ and had a faster rise than decline; the second flare had a slightly more symmetric profile and lasted $\sim 2.2 \mathrm{ks}$. No similar events were found in the second observation.

Extracting the spectra from the time intervals of the flares, we found that the spectral shapes of the two flares are very similar. As a first guess, we assumed that the flare emission is 


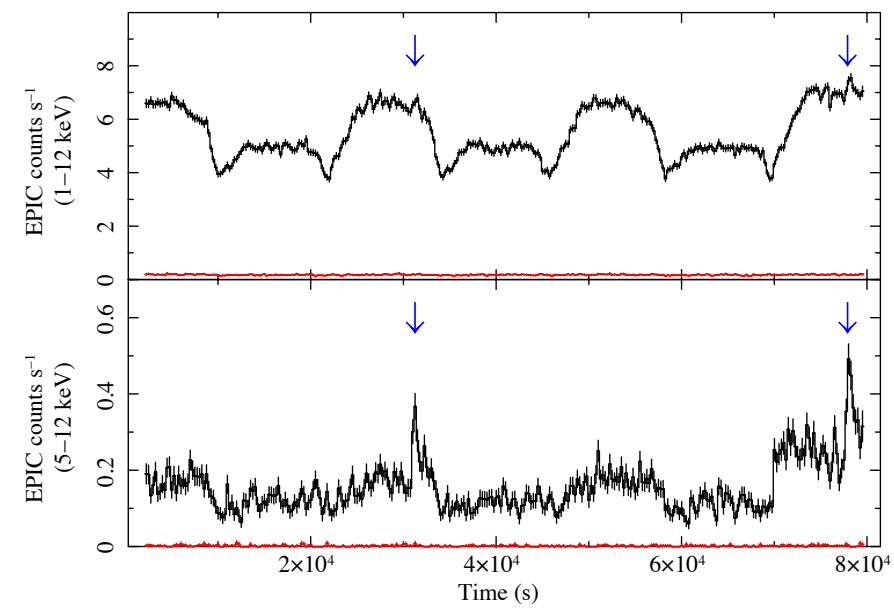

Fig. 4. Light curves of $1 \mathrm{E} 1613$ from the 2016 observation (A) in the $1-12 \mathrm{keV}$ range (top) and in a hard band $(5-12 \mathrm{keV})$ (bottom). The time bin is $250 \mathrm{~s}$. The background contribution is negligible and was not subtracted, but the background level in the proper energy band is shown in red in each panel. The arrows indicate the faint flares (more prominent in the hard band) that are discussed in Sect. 3 .

superimposed on the persistent emission, and we fit the flare spectra with a model consisting of the average spectrum of observation A plus an additional component, either a power law or a blackbody. The fits are essentially equally good; the additional component resembles the hard component of the average spectrum. In the blackbody fit, the temperature converges to a value that is compatible with the value of the hot blackbody in Table 2 . In other words, the spectra of the flares are extremely similar to the spectrum of the persistent emission with an stronger hot component. This can be visually assessed in Fig. 5, where we show a simultaneous fit of the spectra of the average emission and of the two flares where all parameters are tied except for the hot blackbody normalization. The resulting radii are $0.25_{-0.02}^{+0.01}$ and $0.27_{-0.01}^{+0.02} \mathrm{~km}$ for the first and second flare, respectively $\left(\chi_{v}^{2}=1.10\right.$ for 2756 degrees of freedom, d.o.f.). The corresponding luminosities are $(5.7 \pm 0.1) \times 10^{34}$ and $(6.1 \pm 0.1) \times 10^{34} \mathrm{erg} \mathrm{s}^{-1}(0.5-10 \mathrm{keV})$, that is, only $\approx 20-30 \%$ more than the average luminosity (but we note that both events occur at the maximum of the pulse profile, although not exactly at the same phase: they are separated by $\sim 1.9$ cycles).

\section{Discussion}

In 2016, after hibernating for more than a decade, 1E 1613 entered a new phase of magnetar-like activity and produced the first outburst from this source that was monitored intensely and was also followed in hard X-rays and at IR wavelengths (Rea et al. 2016; D'Aì et al. 2016; Tendulkar et al. 2017; Borghese et al. 2018). All studies spotted strong similarities between this unusual source and magnetars, which underlines the possibility that the compact object might be a magnetar (De Luca et al. 2006). The space for a binary system was already narrow in view of the limit on the IR emission that was previously obtained by De Luca et al. (2008), but the ignition of 1E 1613 in the IR that was observed with HST by Tendulkar et al. (2017) strongly argues against the possibility of accretion onto the neutron star. The HST detection implies a brightening $>1.3 \mathrm{mag}$ in the F160W filter band ( $H$ band, $\lambda=1.545 \mu \mathrm{m} ; \Delta \lambda=$ $0.29 \mu \mathrm{m}$ FWHM) and $>1.8 \mathrm{mag}$ in the F110W filter band (wide $J, \lambda=1.15 \mu \mathrm{m} ; \Delta \lambda=0.5 \mu \mathrm{m}$ FWHM). Our (non-simultaneous)

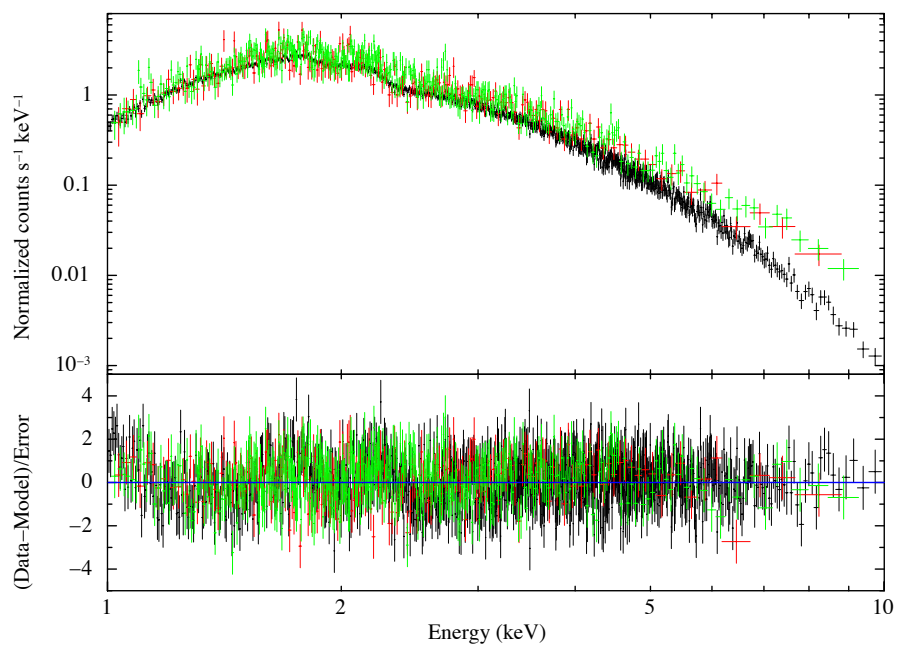

Fig. 5. Spectra of the flares (the data of the first are plotted in red, those of the second in green) compared with that of the average emission (in black). The model fit to the data is the double blackbody. For clarity, we plot only the pn points. Bottom: post-fit residuals.

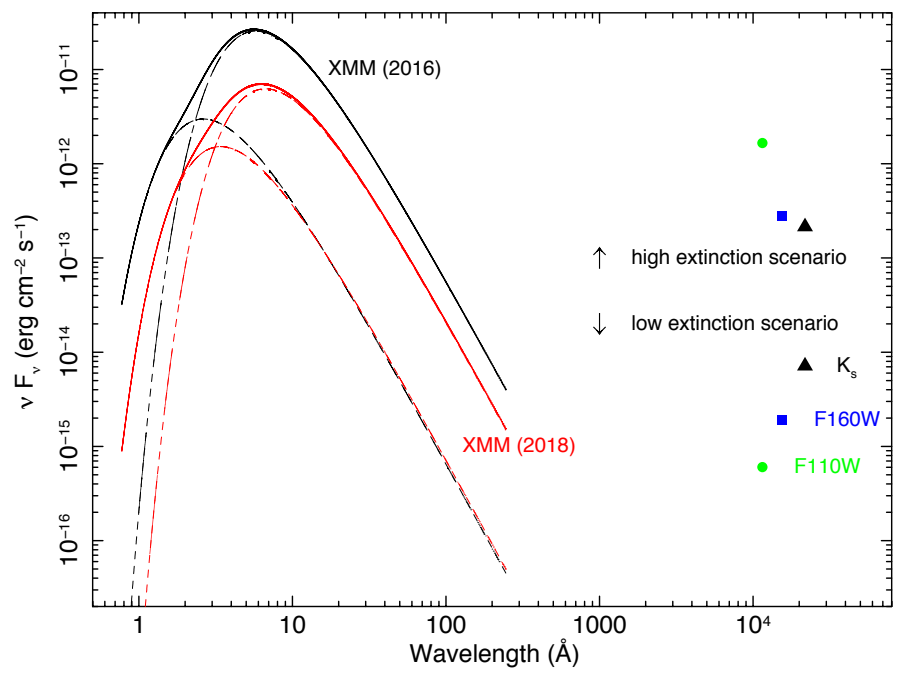

Fig. 6. Spectral energy distribution for 1E 1613 from X-rays to near IR. The XMM-Newton spectral models (double blackbody) are plotted in black for the 2016 data and in red for the 2018 data; the two blackbody components are plotted with the same colors and dashed lines. The black triangles indicate the June 2016 VLT values in the case of low and high extinction ( $A_{V}$ from 3.6 to 36 ). The HST points are from Tendulkar et al. (2017) and the exposures were taken in August 2016 (similar values were found earlier, in July 2016).

VLT observation implies an increase in flux density of at least $\sim 0.5 \mathrm{mag}$ in the $K_{s}$ band. The extinction toward 1E 1613 is uncertain. The dust reddening mapped in its direction is $A_{V}=36$ (Schlafly \& Finkbeiner 2011), and high extinction is indicated also by the colors of field stars (De Luca et al. 2008). On the other hand, much lower values are obtained from optical and IR observations of the SNR and from the $N_{\mathrm{H}}$ measured in the X-ray observations (Oliva et al. 1989; Tendulkar et al. 2017). Following Tendulkar et al. (2017), we assume an $A_{V}$ from 3.6 to 36 but, as they note, a low $A_{V}$ seems more likely because it is suggested by measurements of $1 \mathrm{E} 1613$ and its SNR, while the high values come from analyses of a vast field and unrelated stars. The IRto-X-ray spectral energy distribution of 1E 1613 for the extreme values of $A_{V}$ is shown in Fig. 6 (which, we stress, must be taken 
with caution because the source is variable and the exposures are not simultaneous).

Propeller interaction with a surrounding fallback disk is often proposed as a possible explanation of the slow rotation of 1E 1613 (De Luca et al. 2006; Li 2007; Tong et al. 2016; Ho \& Andersson 2017). Given the lack of constraints on the presence of pulsations in the VLT data, it is hard to tell whether the IR emission is of magnetospheric origin or is due to a disk heated by the enhanced emission of $1 \mathrm{E} 1613$ during the outburst. In the case of low extinction, the $K_{s}$-to-X-ray flux ratio is $\sim 1.8 \times 10^{-5}$, which is similar to the corresponding HST ratios and is in the range observed for magnetars and isolated neutron stars (Mignani 2011; Olausen \& Kaspi 2014). With this assumption, the detection in itself therefore does not support the presence of a fallback disk around the star.

A careful analysis of the source light curves in X-rays revealed the presence of two flares with properties much at variance with those of canonical magnetar bursts. They last much longer ( $\sim 1 \mathrm{ks}$ vs. $0.1-1 \mathrm{~s})$, are less energetic $\left(L \approx 10^{34} \mathrm{erg} \mathrm{s}^{-1}\right.$, exceeding the persistent luminosity by only $\sim 20 \%$, vs. $L \gtrsim$ $10^{38} \mathrm{erg} \mathrm{s}^{-1}$ ), and are softer, with a spectrum quite close to the hotter thermal component of the persistent flux. Because of their long duration, however, their fluence is rather high, $\approx 7 \times 10^{-9}$ and $2 \times 10^{-8} \mathrm{erg} \mathrm{cm}^{-2}$ for the first and second event, respectively, and comparable with standard short burst from magnetars (e.g., Aptekar et al. 2001; Götz et al. 2006; Göğüş et al. 2000).

To the best of our knowledge, an event like this has not been observed before in a magnetar or in other classes of X-ray emitting isolated NSs. However, flares similar to those reported here might well have occurred in other sources and passed unnoticed. The strong similarity of the flare spectrum with the hotter thermal component seen in the persistent emission suggests a common origin. In magnetars, the latter is believed to come from a limited region on the star surface that is heated by dissipative processes. If the flares observed in 1E 1613 do come from the same hot spot, they would be hardly visible in a source with a period $(\approx 0.1-10 \mathrm{~s})$ much shorter than the flare duration $(\approx 1 \mathrm{ks})$, as is the case of all the known X-ray pulsars. In this sense, $1 \mathrm{E} 1613$ is unique because of its period, which is much longer than the flare duration. Further pursuing the idea that the flares originate from a spot on the star surface, they could be produced by some irregularities in the process responsible for the heating. Although the nature of such processes in magnetars is still debated (see, e.g., Turolla et al. 2015; Kaspi \& Beloborodov 2017; Gourgouliatos \& Esposito 2018, for recent reviews), we mention that if the star surface is heated by backflowing currents, an increase in luminosity by $\sim 20 \%$ can easily be explained by a change of the same order in the twist angle or in the potential drop that accelerates the charges (Beloborodov 2009). We also note that the second flare occurred approximately at the same rotational phase as the first, after $\sim 1.9$ cycles. Although with just two events a coincidence cannot be excluded, this might indicate that they came from the same hot spot.

Acknowledgements. The scientific results reported in this article are based on observations obtained with XMM-Newton, an ESA science mission with instruments and contributions directly funded by ESA Member States and NASA.
Based on observations collected at the European Organisation for Astronomical Research in the Southern Hemisphere under ESO programmes 077.D-0764(A) and 297.D-5042(A). PE and ADL acknowledge funding in the framework of the project ULTraS (ASI-INAF contract N. 2017-14-H.0). FCZ is supported by a Juan de la Cierva fellowship.

\section{References}

Aptekar, R. L., Frederiks, D. D., Golenetskii, S. V., et al. 2001, ApJ, 137, 227

Beloborodov, A. M. 2009, ApJ, 703, 1044

Bertin, E., \& Arnouts, S. 1996, A\&AS, 117, 393

Bhadkamkar, H., \& Ghosh, P. 2009, A\&A, 506, 1297

Borghese, A., Coti Zelati, F., Esposito, P., et al. 2018, MNRAS, 478, 741

Caswell, J. L., Murray, J. D., Roger, R. S., Cole, D. J., \& Cooke, D. J. 1975, A\&A, 45, 239

Coti Zelati, F., Rea, N., Pons, J. A., Campana, S., \& Esposito, P. 2018, MNRAS, 474, 961

D’Aì, A., Evans, P. A., Burrows, D. N., et al. 2016, MNRAS, 463, 2394

De Luca, A. 2008, in 40 Years of Pulsars: Millisecond Pulsars, Magnetars and More, eds. C. Bassa, Z. Wang, A. Cumming, \& V. M. Kaspi, AIP Conf. Proc., 983,311

De Luca, A. 2017, in International Conference Physics of Neutron Stars - 2017. 50 years after, St. Petersburg, Russian Federation, eds. G. G. Pavlov, J. A. Pons, P. S. Shternin, \& D. G. Yakovlev, J. Phys. Conf. Ser., 932, 012006

De Luca, A., Caraveo, P. A., Mereghetti, S., Tiengo, A., \& Bignami, G. F. 2006, Science, 313,814

De Luca, A., Mignani, R. P., Zaggia, S., et al. 2008, ApJ, 682, 1185

Devillard, N. 1997, The Messenger, 87, 19

Esposito, P., Turolla, R., De Luca, A., et al. 2011, MNRAS, 418, 170

Esposito, P., Rea, N., \& Israel, G. L. 2018, in Timing Neutron Stars: Pulsations, Oscillations and Explosions, eds. T. Belloni, M. Mendez, \& C. Zhang (Berlin: Springer), ASSL, in press [arXiv:1803.05716]

Gabriel, C., Denby, M., Fyfe, D. J., et al. 2004, in Astronomical Data Analysis Software and Systems (ADASS) XIII, eds. F. Ochsenbein, M. G. Allen, \& D. Egret (San Francisco, CA: ASP), ASP Conf. Ser., 314, 759

Götz, D., Mereghetti, S., Molkov, S., et al. 2006, A\&A, 445, 313

Gourgouliatos, K. N., \& Esposito, P. 2018, in The Physics and Astrophysics of Neutron Stars, eds. L. Rezzolla, P. M. Pizzochero, D. I. Jones, N. Rea, \& I. Vidaña (Basel: Springer Nature Switzerland), 457, 57

Göğüş, E., Woods, P. M., Kouveliotou, C., et al. 2000, ApJ, 532, L121

Ho, W. C. G., \& Andersson, N. 2017, MNRAS, 464, L65

Kaspi, V. M., \& Beloborodov, A. M. 2017, ARA\&A, 55, 261

Lenzen, R., Hartung, M., Brandner, W., et al. 2003, in Instrument Design and

Performance for Optical/Infrared Ground-based Telescopes, eds. M. Iye, \& A. F. M. Moorwood (Bellingham: SPIE), SPIE Conf. Ser., 4841, 944 Li, X. 2007, ApJ, 666, L81

Liu, X. W., Xu, R. X., van den Heuvel, E. P. J., et al. 2015, ApJ, 799, 233

Mignani, R. P. 2011, AdSpR, 47, 1281

Molendi, S., \& Gastaldello, F. 2009, A\&A, 493, 13

Olausen, S. A., \& Kaspi, V. M. 2014, ApJ, 212, 6

Oliva, E., Moorwood, A. F. M., \& Danziger, I. J. 1989, A\&A, 214, 307

Pizzolato, F., Colpi, M., De Luca, A., Mereghetti, S., \& Tiengo, A. 2008, ApJ, 681,530

Popov, S. B., Kaurov, A. A., \& Kaminker, A. D. 2015, PASA, 32, e018

Rea, N., Borghese, A., Esposito, P., et al. 2016, ApJ, 828, L13

Rousset, G., Lacombe, F., Puget, P., et al. 2003, in Adaptive Optical System Technologies II, eds. P. L. Wizinowich, \& D. Bonaccini, (Bellingham: SPIE), SPIE Conf. Ser., 4839, 140

Schlafly, E. F., \& Finkbeiner, D. P. 2011, ApJ, 737, 103

Strüder, L., Briel, U., Dennerl, K., et al. 2001, A\&A, 365, L18

Tendulkar, S. P., Kaspi, V. M., Archibald, R. F., \& Scholz, P. 2017, ApJ, 841, 11

Tong, H., Wang, W., Liu, X. W., \& Xu, R. X. 2016, ApJ, 833, 265

Tuohy, I., \& Garmire, G. 1980, ApJ, 239, L107

Turner, M. J. L., Abbey, A., Arnaud, M., et al. 2001, A\&A, 365, L27

Turolla, R., Zane, S., \& Watts, A. L. 2015, Rep. Progr. Phys., 78, 116901

Wilms, J., Allen, A., \& McCray, R. 2000, ApJ, 542, 914 